\title{
Increased locomotor activity via regulation of GABAergic signalling in foxp2 mutant zebrafish-implications for neurodevelopmental disorders
}

\author{
Teresa M. Lüffe ${ }^{1}$, Andrea D’Orazio ${ }^{1}$, Moritz Bauer ${ }^{1}$, Zoi Gioga ${ }^{1}$, Victoria Schoeffler ${ }^{1}$, Klaus-Peter Lesch (iD ${ }^{2,3,4}$, Marcel Romanos ${ }^{1}$, \\ Carsten Drepper (iD ${ }^{1}$ and Christina Lillesaar (D) ${ }^{1 凶}$
}

(C) The Author(s) 2021

\begin{abstract}
Recent advances in the genetics of neurodevelopmental disorders (NDDs) have identified the transcription factor FOXP2 as one of numerous risk genes, e.g. in autism spectrum disorders (ASD) and attention-deficit/hyperactivity disorder (ADHD). FOXP2 function is suggested to be involved in GABAergic signalling and numerous studies demonstrate that GABAergic function is altered in NDDs, thus disrupting the excitation/inhibition balance. Interestingly, GABAergic signalling components, including glutamatedecarboxylase 1 (Gad1) and GABA receptors, are putative transcriptional targets of FOXP2. However, the specific role of FOXP2 in the pathomechanism of NDDs remains elusive. Here we test the hypothesis that Foxp2 affects behavioural dimensions via GABAergic signalling using zebrafish as model organism. We demonstrate that foxp2 is expressed by a subset of GABAergic neurons located in brain regions involved in motor functions, including the subpallium, posterior tuberculum, thalamus and medulla oblongata. Using CRISPR/Cas9 gene-editing we generated a novel foxp2 zebrafish loss-of-function mutant that exhibits increased locomotor activity. Further, genetic and/or pharmacological disruption of Gad1 or GABA-A receptors causes increased locomotor activity, resembling the phenotype of foxp2 mutants. Application of muscimol, a GABA-A receptor agonist, rescues the hyperactive phenotype induced by the foxp2 loss-of-function. By reverse translation of the therapeutic effect on hyperactive behaviour exerted by methylphenidate, we note that application of methylphenidate evokes different responses in wildtype compared to foxp2 or gad $1 b$ loss-of-function animals. Together, our findings support the hypothesis that foxp2 regulates locomotor activity via GABAergic signalling. This provides one targetable mechanism, which may contribute to behavioural phenotypes commonly observed in NDDs.
\end{abstract}

Translational Psychiatry (2021)11:529; https://doi.org/10.1038/s41398-021-01651-w

\section{INTRODUCTION}

Ever larger and improved genetic studies have enabled substantial progress in the discovery of risk genes for neurodevelopmental disorders (NDDs), such as autism spectrum disorders (ASD) and attention-deficit/hyperactivity disorder (ADHD). However, the involvement of most of the identified risk genes in the pathomechanism remains elusive and requires further extensive functional investigations. Recently, a genome-wide association study of ADHD highlighted 12 loci with genome-wide significance [1]. One of the loci, located on chromosome 7, harbours the gene FOXP2, whose possible role in NDDs is substantiated by several studies $[2,3]$.

FOXP2 belongs to the P subfamily of FOX transcription factors, which are recognised by a highly conserved DNA-binding motif termed winged helix or forkhead domain and by additional subfamily-characteristic domains. It possesses domains involved in homo- and/or hetero-dimerisation, primarily with FOXP1 and FOXP4, as well as in interactions with other proteins, which allows both transcriptional repression and activation in a contextdependent manner $[4,5]$.

In human, FOXP2 is predominantly expressed in the central nervous system (CNS) with prominent presence in the deep layers of the developing cortical plate, the basal ganglia, the thalamus, the inferior olive and the cerebellum [6]. Apart from humans, Foxp2/foxp2 expression has been described for additional mammalian species as well as for birds, reptiles, amphibians and fish, including zebrafish (Danio rerio) [7-17]. These studies revealed a highly concordant localisation within the CNS, which coincides with regions involved in motor functions and therefore suggest a functional conservation of Foxp2. The CNS expression of Foxp2/foxp2 starts during embryonic development and continues postnatally into adulthood and thus indicates a role both in development and maintenance of the CNS. Accordingly, critical functions of Foxp2 in cell differentiation, neurite outgrowth, dendrite morphogenesis, axon guidance and synaptic plasticity have been demonstrated [18-28].

\footnotetext{
${ }^{1}$ Center of Mental Health, Department of Child and Adolescent Psychiatry, University Hospital of Würzburg, Würzburg, Germany. ${ }^{2}$ Center of Mental Health, Division of Molecular Psychiatry, University of Würzburg, Würzburg, Germany. ${ }^{3}$ Laboratory of Psychiatric Neurobiology, Institute of Molecular Medicine, I.M. Sechenov First Moscow State Medical University, Moscow, Russia. ${ }^{4}$ Department of Neuroscience, School for Mental Health and Neuroscience (MHeNS), Maastricht University, Maastricht, The Netherlands. email: Lillesaar_C@ukw.de
} 
The biological pathways underlying Foxp2 deficiency-induced behavioural phenotypes are still largely unknown. However, experimental evidence indicates that altered GABAergic signalling may play a pivotal role in this context $[23,29]$. Thus, Foxp2 is expressed by striatal medium spiny neurons (MSNs) [28], a class of GABAergic neurons that accounts for the majority of the neurons in striatum. Upon Foxp2 impairment, the neurite morphology of these neurons is altered $[19,28]$. Further, in Foxp2 loss-of-function mice, an increased inhibitory presynaptic strength of the striatal direct pathway MSNs due to increased GABA release was observed [29]. This phenotype was accompanied by increased expression of Glutamate-decarboxylase 1 (Gad1, also known as Gad67). In addition, Foxp2 transcriptional targets include genes involved in GABAergic neurotransmission, such as Gad1, Gad2 and GABA receptor subunits [5, 28].

Neuronal network activity, such as in the cortico-striatal circuitry, is controlled by a tightly regulated interaction between excitation (E) and inhibition (I). Under physiological conditions, a definite E/I ratio is maintained via balanced synaptic communication between glutamatergic and GABAergic neurons [30, 31], resulting in the so-called ' $E / I$ balance'. Emerging evidence indicates that the $\mathrm{E} / \mathrm{l}$ balance is dysregulated in NDDs, including ADHD and ASD [32-35]. GABAergic signalling impacts on the developing brain through a variety of regulatory mechanisms ranging from myelination to synaptic communications [36, 37]. These mechanisms program the maturation of interneurons, e.g. MSN, thus allowing strong GABA-mediated feed-forward inhibition to maintain the stability of local networks. The relevance of compromised GABA-mediated inhibition for the aetiology of NDDs is supported by multiple findings both in humans and in model organisms. In human, variants of genes encoding for GABAergic signalling components are associated with ADHD [3841], and mice with genetic disruption of GABAergic signalling components mirror core ADHD symptoms [42-45]. Further, structural brain imaging in ADHD patients shows alterations in regions with prominent GABAergic neuron populations, such as the striatum [46]. Moreover, magnetic resonance spectroscopy revealed altered GABA levels in specific brain regions, including motor cortices, striatum and thalamus of ADHD patients [47-50].

In the present study, we explore the hypothesis that foxp2 regulates behavioural activity via GABAergic signalling. Considering that genetic variants of FOXP2 and/or genes encoding for GABAergic signalling components are associated with ADHD, ASD as well as speech and language disorders [1-3, 38-41], our findings may have implications for NDDs.

\section{MATERIALS AND METHODS}

For detailed descriptions see Supplementary Materials and Methods.

\section{Fish husbandry and embryo preparation}

Experiments were performed on the $A B / A B$ wildtype zebrafish strain (zfin id.: ZDB-GENO-960809-7). Animal handling was performed in accordance with the regulations for animal welfare of the District Government of Lower Franconia, Germany. Larvae were raised in Danieau's solution with or without methylene blue (Cold Spring Harb. Protoc., 2011) at $28^{\circ} \mathrm{C}$ with a light/dark cycle of $14 / 10 \mathrm{~h}$. Determination of developmental stage was according to [51].

\section{Whole-mount RNA in situ hybridisation, immunohistochemistry and image acquisition}

Embryos were raised in Danieau's solution containing $0.2 \mathrm{mM}$ 1-phenyl-2thiourea to suppress pigmentation, manually dechorionated and fixed in $4 \%$ paraformaldehyde (PFA) in phosphate-buffered saline (PBS). A CDNA template for foxp2 covering the last three exons, including the $3^{\prime}$ UTR was cloned and used for in vitro transcription of a DIG-labelled RNA ISH probe (for primers, see Table S1). Whole-mount RNA ISH was performed in accordance with [52]. For two-colour RNA ISH, a mix of DIG-labelled foxp2 and FLUO-labelled gad1a (previously gad67a) RNA probes was applied, and the RNA hybrids were visualised with NBT/BCIP or Fast red, respectively. To further display co-localisation of foxp2 and gad1a double-stained embryos were cryo-sectioned.

To visualise major neurite bundles and tracts, fixed embryos were immunostained with anti-acetylated tubulin (AcTub). Apoptotic cells were identified by application of anti-cleaved caspase 3 (cCasp3). Alexa Fluor 488-conjugated secondary antibodies were used for detection of both primary antibodies. Light and fluorescence images were acquired using a Zeiss Axiophot light microscope, a Leica M205 FA fluorescence microscope or a Zeiss LSM 780 confocal microscope and further processed and analysed using Image J with appropriate plug-ins.

\section{foxp2 and gad1b loss-of-function}

To create a foxp2 loss-of-function, we applied the CRISPR/Cas9 geneediting tool and injected a cocktail of a sgRNA targeting exon 10 of foxp2 (Fig. 1B, Table S1) and the Cas9 protein into the animal pole of fertilised one-cell stage eggs. Injected $F_{0}$ embryos were raised and tested for germline transmission of indel mutations by outcrossing with $A B / A B$ wildtypes and genotyping the offspring. The $F_{1}$ generation was generated by outcrossing positive $F_{0}$ fish with $A B / A B$ wildtypes and used to characterise the indel mutations by Sanger sequencing. Finally, the deletion mutation described in Fig. 1 was selected for subsequent investigations. For the experiments described below, $F_{2}$ or $F_{3}$ embryos and larvae generated by outcrosses of foxp $2^{+/-}$to $A B / A B$ or intercrosses of foxp $2^{+/-}$were used.

A gad1b loss-of-function was created by injections of a splice-inhibiting morpholino oligonucleotide (GeneTools, Table S1) into the animal pole of fertilised one-cell stage eggs. Aberrant splicing was confirmed by RT-PCR and Sanger sequencing at 1 and 5 days post fertilisation (dpf).

\section{Quantitative real-time PCR (qPCR)}

Quantitative real-time RT-PCR (qPCR) was performed on $5 \mathrm{dpf}$ old foxp2 $2^{-1-}$, foxp $2^{+/-}$and foxp $2^{+/+}$siblings. The tail of each embryo was cut for gDNA' extraction and subsequent genotyping. The remaining tissue was used for total RNA extraction and subsequent CDNA synthesis. RNA from 10 pooled embryos was isolated for each genotype, and each target gene (Table S2) was represented by a triplet (technical replicates) of each biological sample $(n=3)$ for each genotype. No RT control (NRT) and no template control (NTC) were included as negative controls. Final quantification and calculation were conducted with the comparative $C_{t}(2-\Delta \Delta C t)$ method using $a c t b 1$ and gapdh as housekeeping genes (Table S2). Significant group differences were determined by applying a one-way ANOVA with the significance level set to 0.05 .

\section{Locomotor tracking}

Locomotion in $5 \mathrm{dpf}$ old larvae was tracked by the semi-automatic system ZebraBox and the commercial software ZebraLab (View Point). Larvae were placed in individual wells of a 12-well plate containing $1 \mathrm{ml}$ of Danieau's solution. The surrounding water was kept constant at $28^{\circ} \mathrm{C}$. Swimming tracks were recorded in the dark with an infrared backlight with a wavelength of $850 \mathrm{~nm}$ by an integrated infrared camera with $30 \mathrm{fps}$. Three activity levels were defined by the following thresholds: inactive, $<0.2 \mathrm{~cm} / \mathrm{s}$; low activity, $>0.2 \mathrm{~cm} / \mathrm{s}$ and $<1 \mathrm{~cm} / \mathrm{s}$; high activity, $>1 \mathrm{~cm} / \mathrm{s}$. Unless stated differently below, larvae were tracked for a total duration of $10 \mathrm{~min}$, with a 5 min habituation and a 5 min test phase. Any larvae exhibiting severe morphological malformations were excluded from the behaviour analysis, and individual wells where misstracking due to technical issues was observed were excluded from the subsequent data analysis. Both exclusion criteria were applied before knowing about the genotype. For final group comparison, only data collected during the test phase was considered for analysis. Locomotor activity was determined by four different parameters: total distance swum, mean velocity during low/high activity or both (total mean velocity), duration of inactivity, low or high activity and the number of events in inactive, low and high activity phase.

\section{Pharmacological treatments}

All substances are listed in Table S3. Glutamate-decarboxylase (Gad) activity was inhibited by the Gad antagonist L-allylglycine (Santa Cruz Biotechnology). A $1 \mathrm{M}$ L-allylglycine stock solution was further diluted in Danieau's solution to a working concentration of $200 \mathrm{mM}$. After tracking 5 dpf old wildtype larvae for $10 \mathrm{~min}$ without treatment (Pre) as described above, $500 \mu \mathrm{l}$ of the total $1 \mathrm{ml}$ Danieau's solution was replaced by the L-allylglycine working solution (final concentration of $100 \mathrm{mM}$ ) or by 
A<smiles>[Si]#C[Te]</smiles>
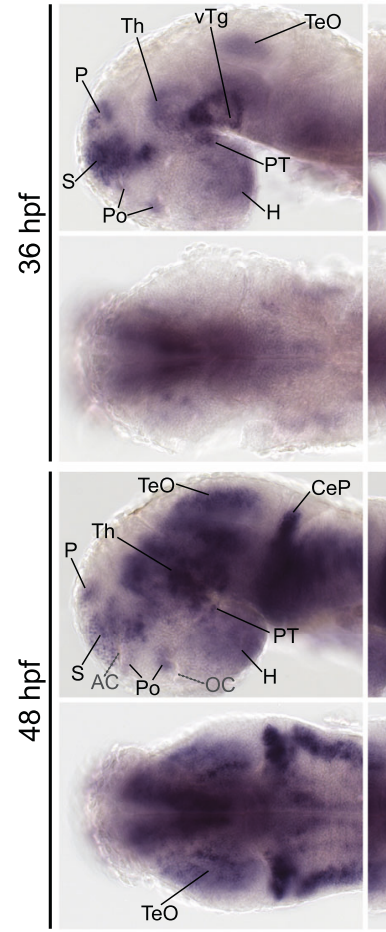

E

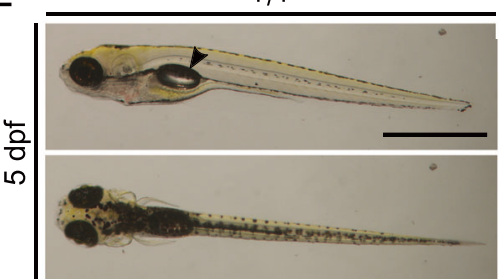

B

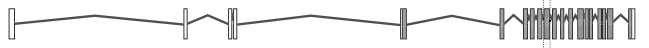

foxp2

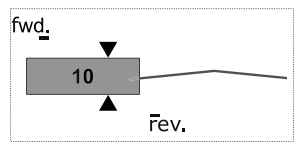

$4: 6451073-6451121$

3. CCTGTACGACACTACTCACT 5 ,

5. TTGAGGGCTGGGGACTGGCCGTTGGACATGCTGTGATGAGTGATGGGTG 3',

3'. AACTCCCGACCCCTGACCGGCAACCTGTACGACACTACTCACTACCCAC 5 ,'

AACT-1-

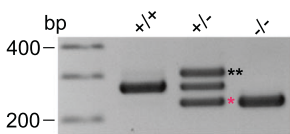

C

foxp2 $2^{+/+}$

MMSPQVIT PQQMQQILQQQVLSPQQLQALLQQQQAVMLQQQHLQEFYKKQQEQLHLQLLQQQ HPGKQAKEQQQQQQQLAAQQLVFQQQLLQMQQLQQQQHLLNMQRQGLLSMPPGPGQPTLPGQ TLPPAGLSPAELQQLWKDVTASHTMEDNGMKHSGLDLSTNNNTSTTSTSNPKASPP ITHHSM SNGQSPALNNNRRESSLHEETAVSHSLYGHGVCKW GCES ICDDFGQFLKHLNNEHALDDRS TAQCRVQMQVVQQLEIQLSKERERLQAMMAHLHMRPSEPKPSPKPLNLVSSVTMSKNLPSIS PPNLPOTPTTPTAPVTPLSOMPOVPNVLSPANVPSMGAMRRRHTDKYSMALSSEIAPNYEFY PPNLPQTPTTPTAPVTPLSQMPQVPNVLSPANVPSMGAMRRRHTDKYSMALSSE IAPNYEFY
KNADVRPPFTYATLIROAIMESSDMOLTLNEIYSWFTRTFAYFRRNAATWKNAVRHNLSLHK KNADVRPPFTYATLIRQAIMESSDMQITLNEIYSWFTRTFAYFRRNAATWKNAVRHNLSLHK
CFVRVENVKGAVWTVDEMEYQKRRSOKITGSPTLVKNLPSSIGYGAALNASLQAALAETTLP LLGNPGLMNSASAMMGASPPVMMSGSPTGLLQGTTHEELNGTLDHLDTNGHSSPGYSPHTHL PPIHVKEEPLNMEDEDCPMSLVTTANHSPELDDDRELEEGNLSEDLE zinc-finger domain leucine-zipper domain winged-helix / forkhead domain

foxp $2^{\%}$ MMSPQVITPQQMQQILQQQVLSPQQLQALLQQQQAVMLQQQHLQEFYKKQQEQLHLQLLQQQ HPGKQAKEQQQQQQQLAAOOLVFOOOLLOMOOLOOQOHLLNMOROGLLSMPPGPGOPTLPGQ TLPPAGLSPAELQQLWKDVTASHTMEDNGMKHSGLDLSTNNNTSTTSTSNPKASPP STTTGE RAHCMKRPQCHTPSMATECASGQDVKVFAMTLDSF ${ }^{*}$

D

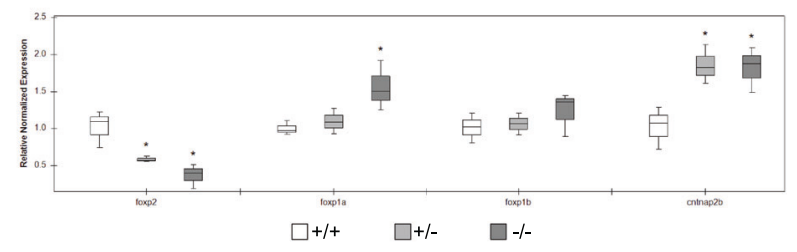

$+/-$
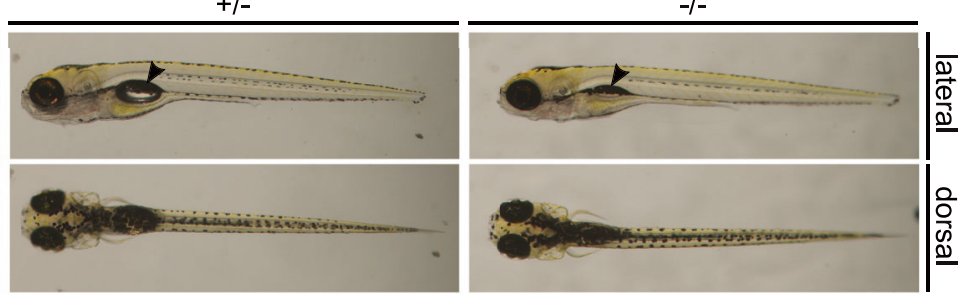

Fig. 1 Expression of foxp2 in the developing CNS of zebrafish and generation of a foxp2 knock-out line using CRISPR/Cas9. A Wholemount RNA in situ hybridisation (ISH) in the developing zebrafish. Anterior is to the left. Abbreviations are listed in Table S4. Arrowheads indicate distinct foxp2-positive populations in the MO. Scale bars: $100 \mu \mathrm{m}$. B foxp2 exon-intron structure with coding (grey) and non-coding exons (white). The sgRNA (pink) targeting foxp2 exon 10 and the primer binding sites for genotyping PCR are indicated. The CRISPR/Cas9induced double-strand break (black triangle) caused a $40 \mathrm{bp}$ deletion represented by a PCR product of 228 bp (pink asterisk) in foxp2 ${ }^{+/-}$and foxp $2^{-1-}$. A third band of $\sim 320 \mathrm{bp}$ corresponds a heterodimer of wildtype and mutated PCR product (black asterisks). C Predicted amino acid sequence of the wildtype (top) and mutated allele (bottom). The frameshifted sequence (black box) is interrupted by a premature stop codon (pink asterisk) N-terminal of the zinc-finger domain. D Relative normalised expression of foxp2, foxp1a, foxp1b and cntnap2b in foxp2 ${ }^{+/+}$(white), foxp2 $2^{+/-}$(light grey) and foxp2 ${ }^{-1-}$ (dark grey) based on qPCR. ${ }^{*} P<0.05$. E Live images of $5 \mathrm{dpf}$ old foxp2 $2^{+/+}$, foxp2 $2^{+/-}$and foxp2 $2^{-1-}$. Anterior is to the left. foxp $2^{-1-}$ show alterations in the development/inflation of the swim bladder (black arrow) in $20 \%$ of the cases. Scale bar, 1 mm.

Danieau's solution without any added substance. After an incubation period of $1 \mathrm{~h}$, the locomotor activity was recorded once per $\mathrm{h}$ for $10 \mathrm{~min}$ with $5 \mathrm{~min}$ habituation and $5 \mathrm{~min}$ test periods over a total period of $8 \mathrm{~h}$ (Post). $10 \mathrm{mM}$ of the GABA-A-receptor (GABA-A-R) antagonist SR-95531 (gabazine) (Thermo Fisher Scientific) was injected into the yolk of fertilised one-cell stage wildtype zebrafish eggs as described elsewhere [53]. At 5 $\mathrm{dpf}$ the locomotor activity was tracked. Wildtype larvae were exposed to
$0.1 \mathrm{mM}$ of the GABA-B-receptor (GABA-B-R) antagonist CGP-55845 (Hello Bio) or to Danieau's solution with $0.1 \%$ DMSO (control) for $48 \mathrm{~h}$ (3-5 dpf). At $5 \mathrm{dpf}$, both solutions were replaced with Danieau's solution without any added substance and locomotion was recorded. $3 \mathrm{dpf}$ old foxp2 ${ }^{+/+}$and foxp $2^{+/-}$siblings were bathed in $25 \mathrm{ml}$ of $0.05 \mathrm{mM}$ muscimol (Merck KGaA) diluted in Danieau's solution or in Danieau's solution only (control) until 5 $\mathrm{dpf}$. During the incubation period, the solution was replaced daily. At $5 \mathrm{dpf}$, 
when locomotion was recorded, it was exchanged for Danieau's solution without any added substance. An $8 \mathrm{mM}$ stock solution of the psychostimulant methylphenidate ( $\mathrm{MPH}$, Merck KGaA) was further diluted to a working concentration of $0.024 \mathrm{mM}$ in Danieau's solution. $5 \mathrm{dpf}$ old larvae were tracked in $1 \mathrm{ml}$ Danieau's solution for $10 \mathrm{~min}$, comprising a $5 \mathrm{~min}$ habituation and a 5 min test phase (Pre). Afterwards, $500 \mu \mathrm{l}$ Danieau's solution was replaced by $500 \mu \mathrm{l}$ of $0.024 \mathrm{mM} \mathrm{MPH}$ working solution $(0.012 \mathrm{mM}$ final concentration) or $500 \mu \mathrm{l}$ Danieau's solution only (control) [54]. After an incubation period of $1 \mathrm{~h}$, the locomotor activity was recorded again by applying the same protocol (Post).

\section{Data analysis and statistics}

Data analysis was performed in RStudio (RStudio 1.3.959, RStudio Team (2020). RStudio: Integrated Development for R. RStudio, PBC, Boston, MA). Data sets were tested for normal distribution by the Shapiro-Wilk's test and for equality of variances by Levene's test. Group differences were calculated distribution-dependent by unpaired $t$-test or unpaired Wilcoxon sign ranked test for two samples or by one-way ANOVA or Kruskal-Wallis rank-sum test for multiple samples. In case of multiple group comparison, we applied Tukey's HSD post hoc test for parametric and Dunn's post hoc test for non-parametric data. The false discovery rate was controlled by the Benjamini-Hochberg adjustment. The general significance level was defined as 0.05 . To pool or compare behavioural data obtained by two versions of the applied tracking software, data frames were standardised using z-score transformation. Effect sizes were determined by Cliff's delta in the effsize package (Torchiano, 2016; Effsize-a package for efficient effect size computation). Appropriate sample sizes were calculated using the software $G^{*}$ Power3.1.9.4 [55] with $a$ and $\beta$ equals 0.05 .

\section{RESULTS}

\section{foxp2 expression in the developing zebrafish CNS}

We used RNA in situ hybridisation to visualise the spatio-temporal distribution of foxp2 transcripts in zebrafish at embryonic and early larval stages (24-72 $\mathrm{h}$ post fertilisation (hpf)). Anatomical abbreviations are listed in Table S4. Expression of foxp2 is first detectable in the telencephalon (Tel) at $24 \mathrm{hpf}$ (Fig. 1A, Supplementary Fig. $1 \mathrm{~A}-\mathrm{B}$ ) and maintained until $72 \mathrm{hpf}$ (Fig. 1A, Supplementary Fig. $1 C-J, K, M, O, Q)$. During subsequent developmental stages, this pattern is further accompanied by expression in the hypothalamus $(\mathrm{H})$ and the ventral tegmentum (vTg) (Supplementary Fig. 1C, D, K) starting at $30 \mathrm{hpf}$, and in the posterior tuberculum (PT), the thalamus (Th), the preoptic region (Po), the optic tectum (TeO) and the medulla oblongata (MO) starting at 36 hpf (Fig. 1A, Supplementary Fig. 1E-J, O-R). At $72 \mathrm{hpf}$, foxp2 transcripts are present in the inner nuclear layer (INL) and the ganglion cell layer (GCL) of the retina (Supplementary Fig. 1S) and dorsally along the spinal cord (SC) (Supplementary Fig. 1T).

\section{Generation of a foxp2 loss-of-function mutation by CRISPR/ \\ Cas9}

To perform functional studies of foxp2 in zebrafish, we generated a foxp2 mutant line with a CRISPR/Cas9 induced $40 \mathrm{bp}$ deletion (Fig. 1B, Supplementary Fig. 2A). The frame shift-induced premature stop codon disrupts the amino acid sequence $\mathrm{N}$-terminal of the zinc-finger domain (Fig. 1C). The nuclear localisation signals, the leucine-zipper, forkhead and C-terminal binding protein 1 binding domains are located downstream of the induced stop codon. Hence, we expect a complete loss of these domains of the Foxp2 protein. The human R328X truncation mutation, which is situated $\mathrm{N}$-terminal to the zinc-finger domain, creates an unstable FOXP2 gene product that remains in the cytoplasm and lacks DNAbinding and transactivation capacity [56]. Since our mutation and the R328X mutation cause a premature stop codon within the similar gene region we expect similar aberrations for any remaining foxp2 gene product in our mutant.

By real-time quantitative PCR (qPCR) we detected a gene dosedependent reduction of foxp2 transcripts in mutants compared to wildtype siblings (Fig. 1D, Table S5), indicating nonsense-mediated decay. Simultaneously, we revealed an upregulation of foxp1a, while foxp $1 b$ expression was not significantly altered. Further, we found a significant upregulation of cntnap $2 b$, a transcriptional target of Foxp2 [57], thus indicating a foxp2 loss-of-function in our mutant.

Homozygous Foxp2 knock-out mice are postnatal lethal, while heterozygous mutants exhibit a mild to moderate developmental delay [58]. Homozygous alleles for the here reported mutation in zebrafish caused alterations in the development or inflation of the swim bladder in about $20 \%$ of the cases (Fig. 1E). In heterozygous mutants, we observed no gross morphological malformations. We found no significant size differences for any of the evaluated parameters (Supplementary Fig. 2B) and no increased apoptosis in foxp $2^{+/-}$or in foxp $2^{-/-}$at $24 \mathrm{hpf}$ (Supplementary Fig. 2C). However, genotyping of older stages revealed that unlike foxp $2^{+/-}$, foxp $2^{-1-}$ do not reach adulthood.

\section{Homozygous deletion of foxp2 results in disorganised commissures and tracts in zebrafish larvae}

Identification of Foxp2 targets involved in neurite development, together with altered neurite morphology following Foxp2 mutations in animal and cell culture models, suggest a role for Foxp2 in neurite growth and guidance [18, 19, 22, 25, 28]. In zebrafish, the effect of Foxp2 on neuritogenesis is unclear. Earlier investigations reported no effect on axon pathfinding in a zincfinger nuclease-induced foxp2 mutant [59], while another zincfinger nuclease-induced mutation affecting cntnap2 caused delayed commissure formation in zebrafish larvae [60]. To investigate whether the here reported mutation of foxp 2 affects the organisation of main commissures and tracts in the developing brain, we performed anti-acetylated tubulin staining on mutant and wildtype siblings. At 20 and $24 \mathrm{hpf}$, we noted a disorganised appearance of the anterior commissure (AC), the post-optic commissure (POC) and the supra-optic tract (SOT) in foxp $2^{-/-}$, while the situation in foxp2 $2^{+/-}$was unchanged (Supplementary Fig. 2D-E). We observed no qualitative effects on the commissure and tract structures at $28 \mathrm{hpf}$ and $5 \mathrm{dpf}$, which suggests that homozygous foxp 2 mutant alleles induce a delay in the early formation of commissures and tracts, which recovers until $28 \mathrm{hpf}$.

foxp2 deletion increases locomotor activity in zebrafish larvae Foxp2 is implicated in motor functions in mammals as well as in flies. Besides complex motor tasks such as sequenced orofacial movements during speech in humans [61], motor skill learning, vocalisation or vocal imitation in mammals or birds $[24,26,62,63]$ findings in fruit flies have demonstrated a role for Foxp2 in basic motor functions such as locomotion [18]. To investigate whether Foxp2 plays a role in the regulation of basic motor functions in zebrafish larvae, we compared locomotion of $5 \mathrm{dpf}$ old foxp2 mutants and wildtype siblings (Fig. 2A). For both foxp $2^{+/-}$and foxp $2^{-/-}$, we measured a significant increase in locomotor activity displayed as an increased swimming velocity and distance (Fig. $2 \mathrm{~B})$. In addition, the velocity and the duration of "high activity" were significantly increased for both mutant groups (Fig. 2B). Beyond that, foxp $2^{+/-}$mutants displayed a significantly elevated number of "high activity" swimming events (Fig. 2B).

\section{foxp2 is expressed by GABAergic neurons}

Previous studies performed to identify neuronal circuits underlying motor phenotypes in Foxp2 mutants focused predominantly on dopamine-dependent neuromodulation in the striatum or homologous brain regions $[22,64]$. In the striatum, Foxp2 is expressed by GABAergic MSNs [28], which are modulated by dopamine and regulate motor functions [65]. Interestingly, Foxp2 loss-of-function increases striatal direct pathway inhibition through reduced transcriptional repression of Gad1 [29], which indicates a regulation of GABA-mediated inhibition as a functional role of Foxp2. 
A

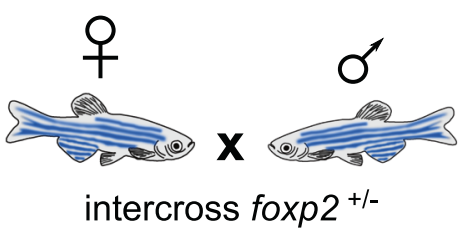

$\stackrel{0}{\text { habituation }} \stackrel{5}{+}$ test $10 \mathrm{~min}$
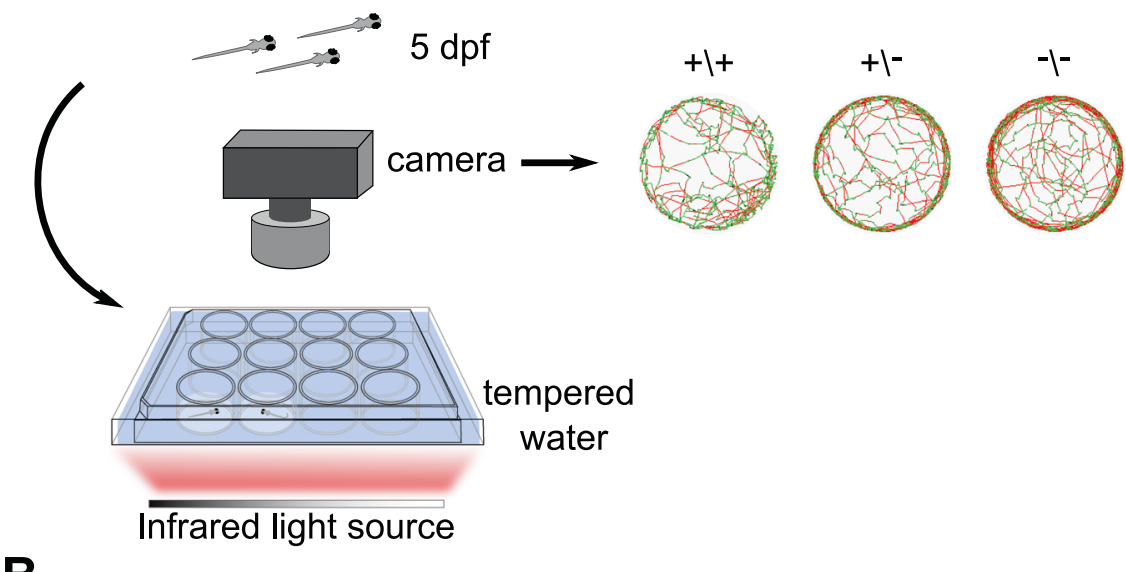

B
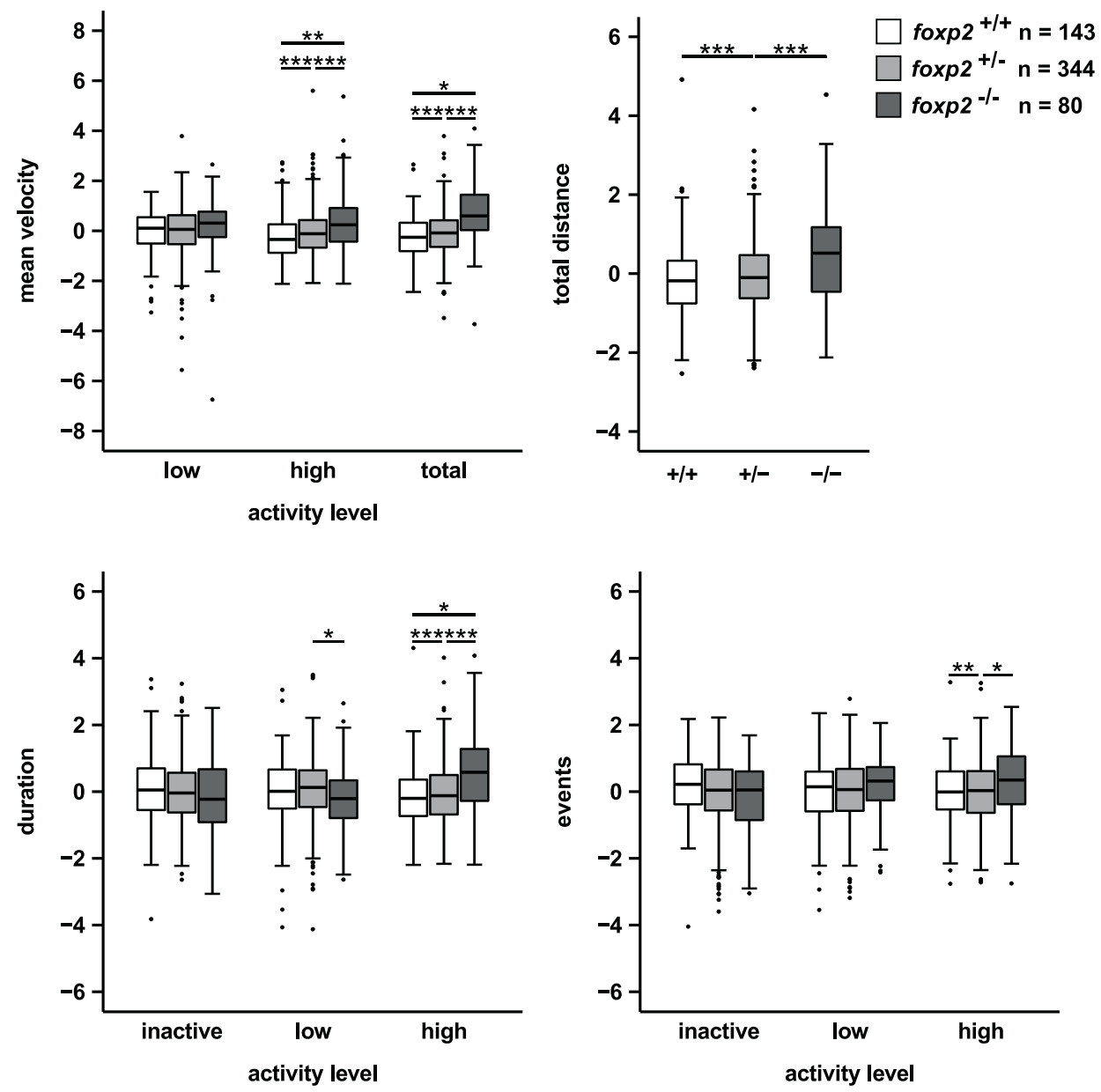

Fig. 2 Increased locomotor activity in larval foxp2 mutants. A Crossing scheme and behavioural setup applied for locomotor tracking in 5 $\mathrm{dpf}$ old foxp $2^{+/+}$, foxp $2^{+/-}$and foxp2 $2^{-1-}$. Circles illustrate representative swim tracks of individual fish with inactive $(<0.2 \mathrm{~cm} / \mathrm{s}$, black), low activity $\left(>0.2 \mathrm{~cm} / \mathrm{s}\right.$ and $<1 \mathrm{~cm} / \mathrm{s}$, green) and high activity $\left(>1 \mathrm{~cm} / \mathrm{s}\right.$, red). B Locomotor activity of foxp $2^{+/+}$(white), foxp2 $2^{+/-}$(light grey) and foxp $2^{-1-}$ (dark grey) analysed for mean velocity (top left) in low or high activity, or combined (total), total distance swum (top right), duration (bottom left) and events (bottom right) of inactivity, low and high activity. Raw data was standardised using $z$-score transformation. ${ }^{*} P<0.05$, ${ }^{* *} P<0.01,{ }^{* * *} P<0.001$. 
To investigate whether foxp2 is expressed by GABAergic neurons of the developing zebrafish brain, particularly in regions involved in motor control, we performed two-colour RNA ISH for foxp2 and gad1a. Since the expression pattern of both GAD1 paralogs, gad $1 a$ and gad1b, are highly similar (Supplementary Fig. $3 \mathrm{~A}-\mathrm{L}$ ), we decided for gad1a as GABAergic marker due to technical reasons. For all three stages examined, foxp2 expression partially overlaps with gad1a expression in the subpallium (S), the preoptic region (Po), the thalamus (Th), the posterior tuberculum (PT) (Fig. 3A, C, E, Supplementary Figs. 4A, C, E and 5A, C, E) and the lateral and dorsal medulla oblongata (MO) (Fig. 3B, D, F, Supplementary Figs. 4B, D, F and 5B, D, F). Co-expression of foxp2 and gad1a was further visualised and confirmed on cryo-sections in the aforementioned regions (Fig. 3G-R, Supplementary Figs. 4G$\mathrm{R}$ and $5 \mathrm{G}-\mathrm{R})$. In addition, later stages display overlapping expression of foxp2 and gad1a in the optic tectum (TeO) and the inner nuclear layer (INL) of the retina (Supplementary Fig. 5A, $\mathrm{C}, \mathrm{G}, \mathrm{H}, \mathrm{K})$. Hence, we confirmed that foxp2 is expressed in a subset of GABAergic neurons in tel-, di-, mes- and rhombencephalon of the developing zebrafish brain with striking incidence for areas involved in motor functions.

\section{Interference with GABAergic signalling alters locomotor activity}

The data described above confirm the presence of foxp2 in GABAergic neurons in developing brain regions involved in motor
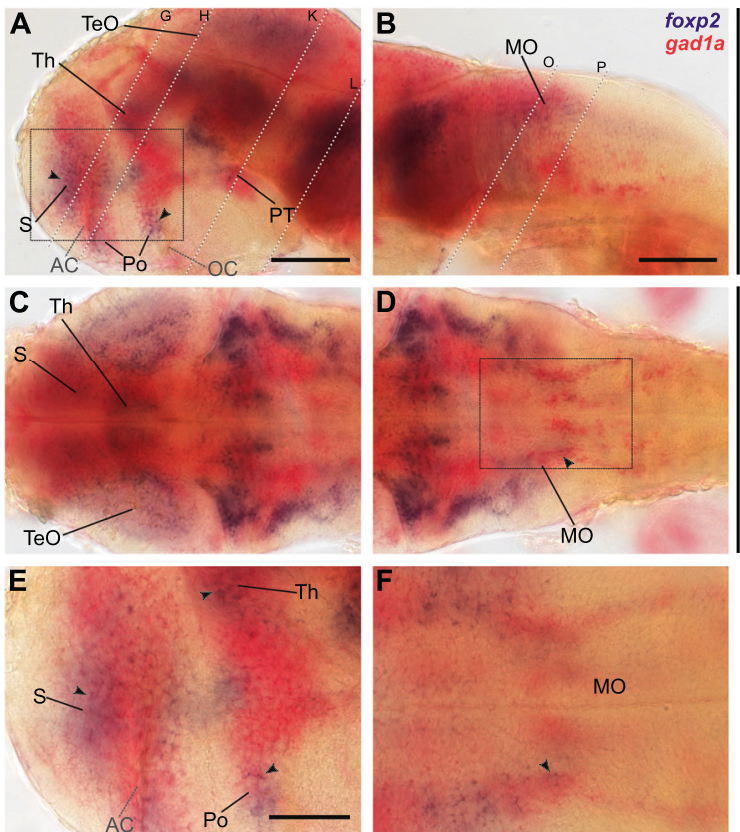
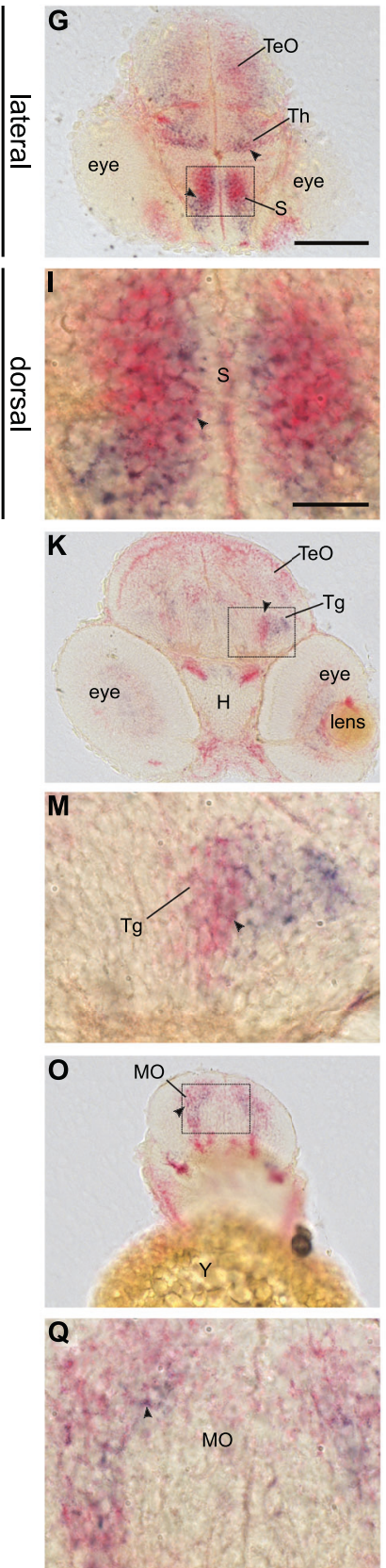
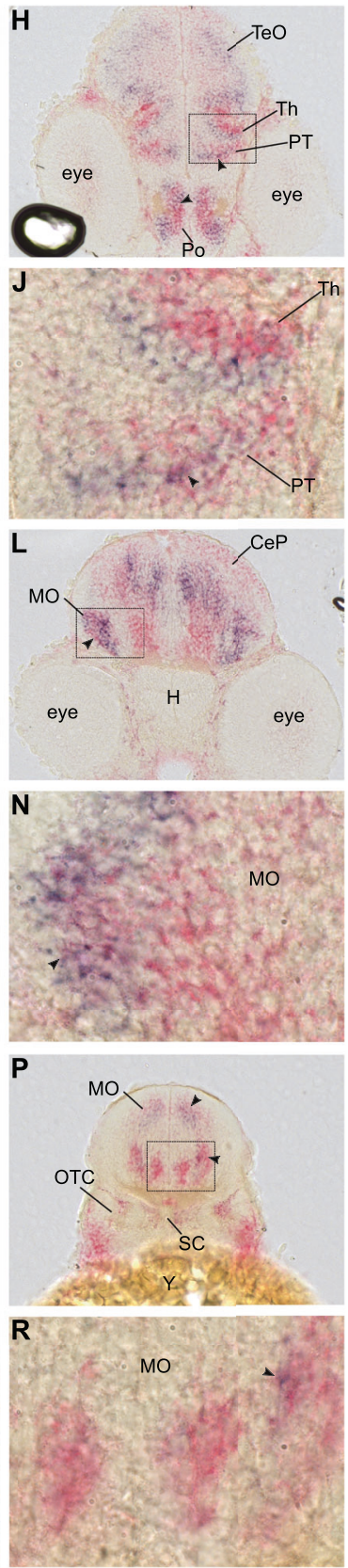

Fig. 3 foxp2 is expressed by a subset of gad1a positive neurons in the developing CNS. Double labelling of foxp2 (blue) and gad1a (red) expression in $48 \mathrm{hpf}$ old wildtype embryos using two-colour RNA ISH. Lateral (A, B, E) and dorsal (C, D, F) overview of embryonic CNS with anterior to the left. E, F Magnifications of boxed areas in A and D. Dashed lines indicate cutting sites for cross-sections displayed in G-R. I, J, M, $\mathbf{N}, \mathbf{Q}, \mathbf{R}$ magnifications of boxed areas in $\mathbf{G}, \mathbf{H}, \mathbf{K}, \mathbf{L}, \mathbf{O}, \mathbf{P}$, respectively. Arrows indicate sites of co-localisation. Abbreviations are listed in Table S4. Scale bars: $100 \mu \mathrm{m}$ (overview), $50 \mu \mathrm{m}$ (magnified images). 
control and show that foxp2 loss-of-function results in increased locomotor activity. Interestingly, impaired Gad1 function is implicated in altered motor function [43, 66]. Hence, we hypothesised that Gad1-activity is important for the regulation of locomotor behaviour in zebrafish larvae. We applied two complementary strategies to interfere with Gad1 activity: a spliceinhibiting morpholino targeting the gad $1 b$ transcript or application of L-allylglycine, a direct Gad antagonist. Targeting gad1b instead of gad1a was motivated by a higher amino acid similarity compared to human GAD1 (Gad1a, 81\%; Gad1b, 84\%; ensembl. org, GRCZ11).

The gad $1 b$ splice-inhibiting morpholino caused retention of intron 8 detected at 1 and $5 \mathrm{dpf}$ and a decreased amount of wildtype transcript at $1 \mathrm{dpf}$ that partly recovered until $5 \mathrm{dpf}$ (Fig. $4 A-B$, Supplementary Fig. 6A). The intron retention induces a frameshift and a subsequent premature stop codon. No gross morphological alterations were observed (Fig. 4C). Size measurements revealed no significant effect on head size or yolk diameter, but a slight reduction in body length (Supplementary Fig. 6B). Unlike foxp2 mutants, gad1b morphants exhibited increased cell death (Supplementary Fig. 6C). However, wildtypes treated with L-allylglycine at $5 \mathrm{dpf}$ showed no apparent changes in size or cell apoptosis (not shown), which may be attributed to the acute treatment strategy later in development.

During locomotor tracking, we found that gad $1 b$ knock-down induces a hyperactive phenotype similar to that of foxp2 mutants (Fig. 4D). Further, we pharmacologically phenocopied this effect in wildtype individuals, however with a stronger effect and in absence of any developmental delay or anatomical alterations through the acute application of the Gad antagonist L-allylglycine (Fig. 4E). Taken together, these results show that transcriptional loss of gad1b or pharmacological blockage of Gad induces hyperactivity, a behavioural phenotype that resembles a genetic loss of foxp2.

We complemented our investigations by inhibiting either GABA-A-receptors (GABA-A-R) or GABA-B-receptors (GABA-B-R) and subsequent locomotor tracking. Interestingly, we measured opposite effects with increased locomotor activity in wildtype individuals treated with the GABA-A-R antagonist SR-95531 (Fig. 4F) and decreased locomotor activity upon exposure to the GABA-B-R antagonist CGP-55845 (Fig. 4G).

To summarise, by targeting different levels of GABAergic signalling, including the gad $1 b$ transcript, the Gad enzymatic activity and two receptors (GABA-A-R and GABA-B-R), we showed that GABAergic signalling plays an important role in the regulation of locomotor behaviour in zebrafish larvae.

\section{Increased locomotor activity in foxp2 mutants is rescued by increased GABA-A-R-mediated inhibition}

Motivated by our qPCR results, showing a significant reduction in the amount of gad $1 b$ transcript in foxp $2^{+/-}$and a tendency to higher gad $1 a$ and gad2 transcript levels in foxp $2^{+/-}$and foxp $2^{-/-}$ (Fig. 5A), we hypothesised that the observed increase in locomotor activity of foxp2 mutants might be caused by an alteration of Gad-regulated GABAergic inhibition. Due to the significant downregulation of gad $1 b$ expression and the consistent phenotype across foxp 2 knock-out and gad1b knock-down, we suggest a reduction of GABAergic inhibition as one possible cause.

To compensate for a potential reduction of GABAergic inhibition in foxp $2^{+/-}$we applied the GABA-A-R agonist muscimol. Under control conditions, we confirmed the previously observed increase in locomotor activity of foxp $2^{+/-}$compared to foxp $2^{+/+}$ (Fig. 5B). However, in response to muscimol, foxp $2^{+/-}$significantly reduced their locomotor activity to a level similar to that of untreated foxp $2^{+/+}$, whereas the locomotor activity of foxp $2^{+/+}$ was not significantly altered compared to the control conditions (Fig. 5B). Hence, we showed that the increased locomotor activity of foxp $2^{+/-}$can be rescued by increasing GABA-A-R-mediated inhibition and that foxp $2^{+/-}$and foxp $2^{+/+}$respond differently to muscimol.

\section{MPH treatment differentially alters locomotor activity in foxp2 mutants and gad1b morphants in comparison to wildtype controls}

Both FOXP2 and GAD1 are identified as potential ADHD risk genes $[1,39]$. Since hyperactivity represents one of the core behavioural endophenotypes in ADHD symptomatology, we tested whether we could rescue the observed hyperactivity of foxp $2^{+/-}$and gad $1 b$ morphants by exposure to methylphenidate (MPH), a wellestablished psychostimulant in ADHD pharmacotherapy [46].

Interestingly, the locomotor hyperactivity of foxp2 ${ }^{+-}$was not affected by MPH treatment (Fig. 5C), whereas in foxp $2^{+/+} \mathrm{MPH}$ increased locomotor activity. We made similar observations in gad1b morphants and wildtype controls. While $\mathrm{MPH}$ induces increased locomotor activity in wildtype controls (Fig. 5D), it does not significantly alter locomotion of gad1b morphants (Fig. 5D). Thus, wildtypes, foxp $2^{+/-}$and gad $1 b$ morphants exhibit a differential response to $\mathrm{MPH}$, suggesting that direct or indirect MPH targets are altered upon foxp2 and gad1b loss-of-function.

\section{DISCUSSION}

Here we explore the hypothesis that Foxp2 and GABAergic signalling are part of a biological network involved in the regulation of behavioural activity. We show that foxp2 loss-offunction results in increased locomotor activity and demonstrate that foxp2 is expressed by GABAergic neurons in several brain regions involved in motor functions. Intriguingly, disruption of Gad1 or GABA-A receptor activity causes hyperactivity, resembling the phenotype observed in our foxp2 mutants. By application of the GABA-A-R agonist muscimol, we are able to rescue the hyperactive phenotype induced by the foxp2 loss-of-function. Together these findings support the hypothesis that foxp2 regulates locomotor activity via GABAergic signalling. This provides one possible mechanism by which behavioural phenotypes, such as altered activity, seen in NDDs may be explained.

By qPCR, we note a significant gene dose-dependent reduction of foxp2 transcripts and a concurrent upregulation of foxp1 transcripts in mutants. Compensatory upregulation of paralogous genes is a well-known phenomenon under knock-out conditions and may account for a milder or even a lack of an expected phenotype [67], especially since Foxp1 and Foxp2 share several transcriptional targets [68]. In addition, recent expression data suggests that Foxp1 and Foxp2 may function as direct regulatory opponents in some neurons [69], whereas providing coordinated regulation in others [68]. Hence the upregulation of foxp $1 a$ (and foxp $1 b$ ), whether due to genetic compensation or de-repression, can cause phenotypic buffering, but may as well be a crucial mechanism in the observed foxp2 mutant phenotype. Since we observed a gene dose-dependent phenotype in expression, morphology and behaviour we conclude, that the upregulation of foxp2 paralogs cannot (fully) compensate for the loss of foxp2. However, we cannot exclude that the phenotypes would be more severe or different in double or triple mutants where additional foxp homologs are disrupted. Besides foxp $1 a$, we found a significant upregulation of cntnap $2 b$ and mef2cb (Supplementary Fig. 7, Table S5), thereby confirming well-known targets of Foxp2 $[19,57]$ also in the zebrafish. Our results indicate that Foxp2 acts as a transcriptional repressor on cntnap $2 b$. A previously published zinc-finger nuclease-induced foxp2 zebrafish mutant rather suggest transcriptional activation of cntnap2b [59]. These seemingly conflicting findings may be explained by a contextdependent function of Foxp2, such as different genetic backgrounds or stage-dependent interaction with distinct transcriptional co-factors. Observations from other model systems show that Foxp2 usually acts as a repressor, but can also activate gene 
A

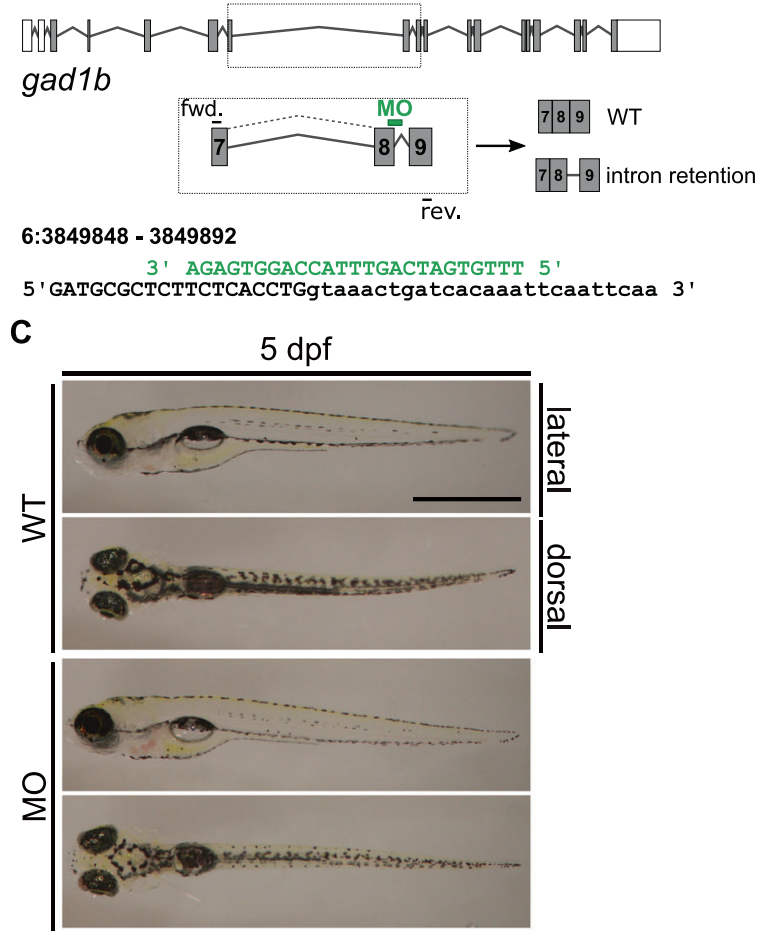

B
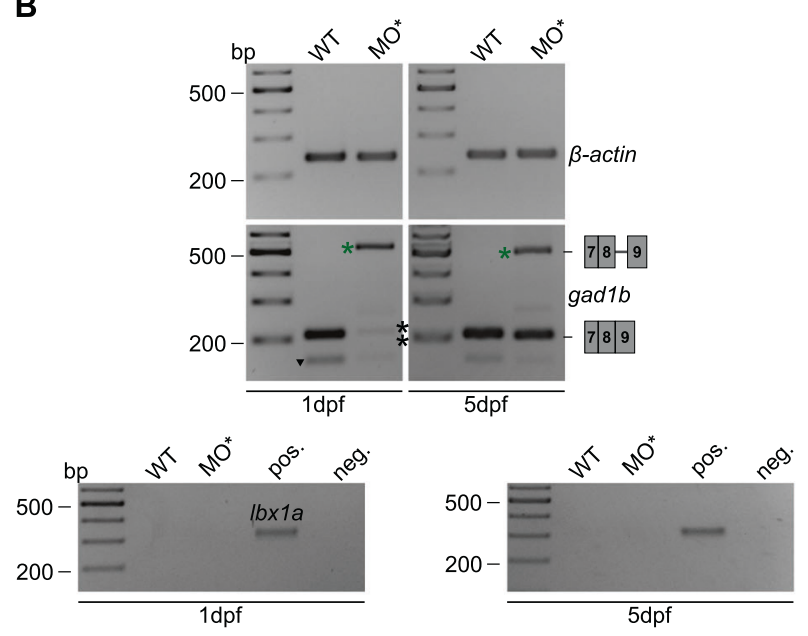

$8-9$

WT 206 MFTYEIAPVFVLMEQLTLKKMREIIGWPNGDGDALFSPG 244 MO* 206 MFTYEIAPVFVLMEQLTLKKMREI IGWPNGDGDALFSPG 244

WT

MO* klitnsiqftictgyiliqnttyycknysplyyni* $\begin{array}{lll}\text { WT } 245 \text { GAISNMYSVMVARYKYFPEVKTKGMSAAPRLVLFTSEH } & 282 \\ \text { MO* } 245 \text { - } & \end{array}$
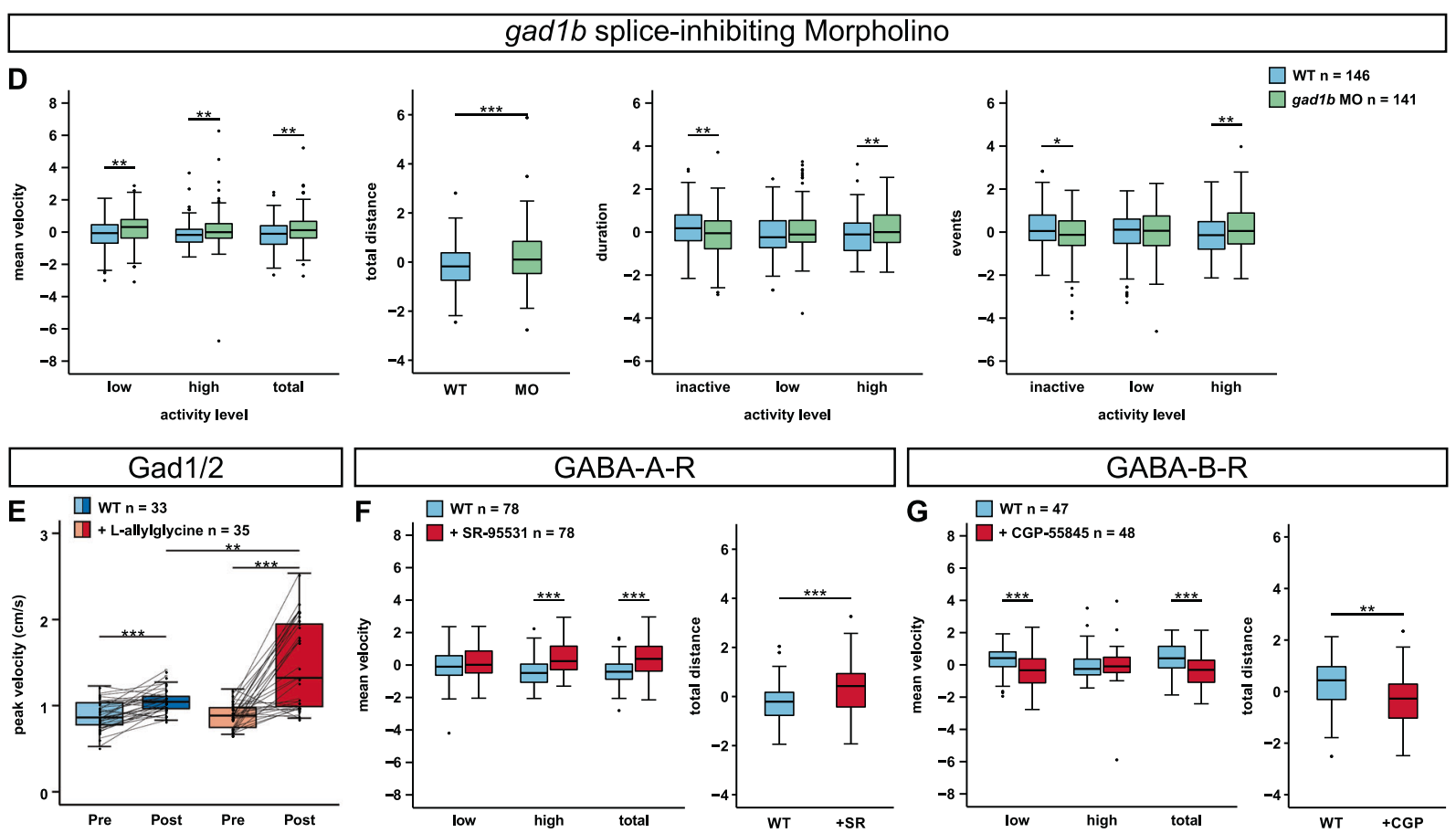

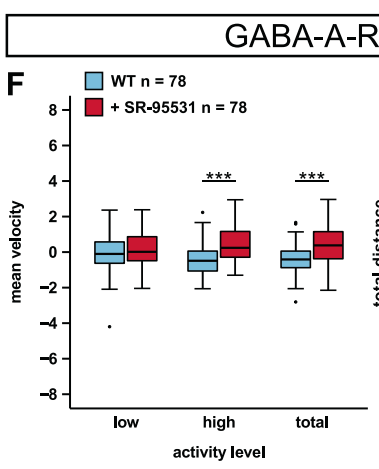

expression [4, 5]. Finally, many of the potential Foxp2 targets tested by qPCR in the present study (Supplementary Fig. 7, Table S5) and which are previously identified NDDs risk genes $[1,3,54,70,71]$ exhibit increased expression levels, although only a few of them reach statistical significance. This supports the suggestion that Foxp2 is a central actor in a molecular network affected in multiple NDDs.

The aforementioned zinc-finger nuclease-induced foxp2 zebrafish mutant exhibited a normal morphological appearance [59]. The mutant was not examined for any behavioural phenotypes. In our study, we similarly conclude that the macroscopic morphology is normal, except for a loss of the swim bladder in about $20 \%$ of the foxp $2^{-\prime-}$. However, our analysis of major neurite bundles in the brain revealed a transient disorganised appearance at early stages. This suggests a rapid recovery in neurite growth and a narrow critical window when foxp 2 influences major neurite growth. Notably, cntnap $2 a$ and $2 b$ mutants display a similar phenotype that recovers with progressing development [60]. Hence, altered expression of cntnap2b (and cntnap2a) might be responsible for the transiently disorganised neurite bundle structure in the here presented foxp $2^{-1-}$ mutants. Our current analysis is not detailed enough to allow us to conclude about the 
Fig. 4 Effects of altered GABAergic signalling on locomotor activity in $\mathbf{5}$ dpf old zebrafish larvae. A gad1b exon-intron structure with coding (grey) and non-coding exons (white). gad1b splice-inhibiting morpholino (MO, green) and corresponding primer binding sites are indicated. Binding of the gad1b-MO causes retention of intron 8. B, top and centre The reduction of the gad1b wildtype transcript at $1 \mathrm{dpf}$ (black asterisks, $208 \mathrm{bp}$ ) and the presence of the misspliced transcript at 1 and $5 \mathrm{dpf}$ (green asterisk, $500 \mathrm{bp}$ ) was verified by RT-PCR. A primer off-target (black triangle) was detected below the wildtype gad1b transcript. Control PCRs on beta-actin (above, 239 bp) and lbx $1 a$ (below, $353 \mathrm{bp}$ ) confirmed comparable cDNA levels and absent genomic DNA contamination for all samples. B, bottom The predicted amino acid sequence of the misspliced gad $1 b$ transcript suggests a premature termination of translation in gad $1 b$ intron 8 (asterisk). C Overviews of a 5 $\mathrm{dpf}$ old gad $1 b$ morphant (MO) and an uninjected control larva (WT). Scale bar, $1 \mathrm{~mm}$. D Locomotor activity of $5 \mathrm{dpf}$ old gad1b morphant larvae (MO, green) compared to uninjected controls (WT, light blue). Locomotor activity was assessed by mean velocity in low or high activity, or combined (total), total distance swum, duration or events of inactivity, low and high activity. E Maximum mean velocity (of 5 min) reached within $8 \mathrm{~h}$ of tracking (peak velocity in $\mathrm{cm} / \mathrm{s}$ ) after incubation (Post) in $100 \mathrm{mM}$ Gad-inhibitor L-allylglycine (dark red) or Danieau's solution (control, dark blue). Maximum mean velocity (of $5 \mathrm{~min}$ ) of the same individuals before incubation in L-allylglycine (Pre, light red) or Danieau's solution (Pre, light blue). Line plot displays activity changes of individual larvae. $\mathbf{F}$ Locomotor activity of 5 dpf old wildtype larvae injected with 10 mM GABA-A-receptor antagonist SR-95531 (red) or water (light blue) at one-cell stage. G Effect of 0.1 mM GABA-B-receptor antagonist CGP55845 on locomotor activity of $5 \mathrm{dpf}$ old wildtype larvae (red) in comparison to Danieau's solution incubated controls (light blue). D, F, G Raw data was standardised using $z$-score transformation. ${ }^{*} P<0.05,{ }^{*} P<0.01,{ }^{*}{ }^{*} P<0.001$.

fine organisation of neurites and synapses, but is an interesting path for future investigations, in particular with respect to foxp2positive populations of GABAergic neurons.

The spatio-temporal expression analysis of foxp2 confirms previous findings [7] and reveals a conform picture of foxp2 transcript distribution in teleost fish $[10,13]$ and a comparable distribution of FOXP2/Foxp2 for mammals, amphibians, reptiles and birds $[6,8,9,11,12,14-17]$. Thus, our findings support the notion of an evolutionary conserved expression of FOXP2/Foxp2/ foxp 2 in the vertebrate brain. Of particular interest in our foxp 2 expression analysis is the high resemblance to the distribution of GABAergic neuron populations [72]. By performing a double RNA ISH, we show that foxp2 and gad1a overlap and co-localise especially in distinct areas essential for motor functions [65]. These observations are of major interest with regard to motor deficits observed in various Foxp2 mutant lines [58, 62] and propose altered GABAergic signalling as a crucial factor for further investigations on motor impairments in foxp2/Foxp2 deficient animals and FOXP2-associated human disorders.

Aberrations in GABAergic signalling are implicated in NDDs. Accordingly, a familial duplication of a cluster of four $G A B A_{A}$ receptor subunit genes segregates with multiple NDDs [38], polymorphisms in GAD1 are associated in particular with the hyperactive/impulsive domain in children with ADHD [39], variants of the GABA-transporter gene GAT1 (SLC6A1) correlates with the risk for ADHD in a case-control study [40] and multiple genetic variants with known direct relation to GABAergic or glutamatergic signalling are associated to symptom severity in ADHD [41]. Further, brain imaging consistently shows alterations in brain regions with substantial GABAergic neuron populations in ADHD, e.g. prefrontal cortex, basal ganglia and cerebellum [46]. Magnetic resonance spectroscopy revealed altered levels of GABA in the prefrontal, anterior cingulate, motor and primary somatosensory cortices as well as in the basal ganglia in ADHD patients [47-49]. In a study of monozygotic twins discordant for ADHD, brain imaging combined with epigenetic evaluations demonstrated alterations in striatum and cerebellum correlating with differential methylation of GABAergic genes [50]. Similarly, animal models support the notion of GABA playing a yet underrated role in the pathophysiology of NDDs. Genetic mouse models with impaired function GABAergic signalling components mirror core ADHD symptoms such as hyperactivity, impaired sustained attention and increased impulsivity [42-45]. Interestingly, hyperactivity in the Gat1 knockout mice was rescued by stimulant application [45]. Further, Cdh13 knock-out mice display ADHD-like phenotypes and $\mathrm{Cdh} 13$ localises to GABAergic neurons [34, 73]. Both mouse Cntnap2 and zebrafish cntnap $2 a / 2 b$ loss-of-function exhibit loss of GABAergic neurons and display hyperactivity $[60,74]$.

The observations described above, combined with the colocalisation of gad1a and foxp2 in regions involved in motor control, raise the question whether FOXP2/Foxp2 may be part of a biological network, which regulates GABAergic signalling and thereby motor functions affected in NDDs. In the present study we address this question and provide two major findings. Firstly, interference with gad1b, Gad or GABA-A-Rs results in an increased locomotor activity similar to what we find after foxp2 impairment, thus we demonstrate that GABAergic signalling influences locomotor activity in zebrafish. Secondly, the hyperactive phenotype of foxp2 mutants is rescued by muscimol-induced GABA-A-R activation. This reveals that the hyperactivity induced by foxp2 impairment is, at least partly, caused by a deficit in GABAergic signalling that can be compensated for by a direct activation of GABA-A-Rs. The opposite effects on locomotor activity upon application of GABA-A-R or GABA-B-R antagonists can be explained by the disparate synaptic localisation and downstream functions of the two receptor classes.

It is well established that pharmacological substances used to treat $\mathrm{ADHD}$ such as methylphenidate (MPH) primarily target catecholaminergic neurotransmission [46]. However, effects of MPH on GABAergic signalling have been noted both in humans and in animal models [75-77]. In addition, substances with known GABAergic effects may be beneficial in treatment of ADHD symptoms [33]. We therefore tested the impact of MPH on our foxp2 and gad1b loss-of-function situations. As expected, we found that the activity level of wildtypes is increased in response to $\mathrm{MPH}$. Similar responses are reported in multiple experimental situations and are attributed to the stimulant effect of MPH [78]. In contrast, neither foxp $2^{+/-}$nor gad1b morphants responded to $\mathrm{MPH}$. This suggests an altered function of direct or indirect MPH target(s) upon foxp2 or gad1b loss-of-functions. Interestingly, activation of $D_{1}$ or $D_{2}$ receptors increases or decreases movement initiation, respectively, in zebrafish larvae [79]. Notably the $D_{1}$ receptor encoding gene is a proposed transcriptional target of Foxp2 [64, 80] and both are expressed by GABAergic forebrain neurons, which exhibit altered function upon Foxp2 loss-offunction [29]. Hence, we speculate that the reduced MPH sensitivity of foxp 2 mutants and gad $1 b$ morphants may be caused by reduced $D_{1}$ receptor levels in foxp 2 mutants and/or altered downstream signalling capacity through impaired GABA synthesis in both foxp2 mutants and gad1b morphants.

In summary, we conclude that Foxp2 regulates GABAergic signalling and that this is a crucial mechanism for the regulation of locomotor activity in zebrafish larvae. Further, we propose that inhibition of downstream targets is mediated by GABA-A-R activation. Currently we can only speculate about the identity of the involved neuronal circuitries. Previous investigations in zebrafish showed co-expression of foxp2 and a $d / x 5$ and dlx6 reporter transgene marking striatal basal ganglia [7]. Interestingly, Dlx genes are essential for the differentiation of GABAergic forebrain neurons [81], and here we noted co-localisation of foxp2 and gad1a in the ventral forebrain. Together with previously reported alterations in striatal GABAergic inhibition as well as 
A

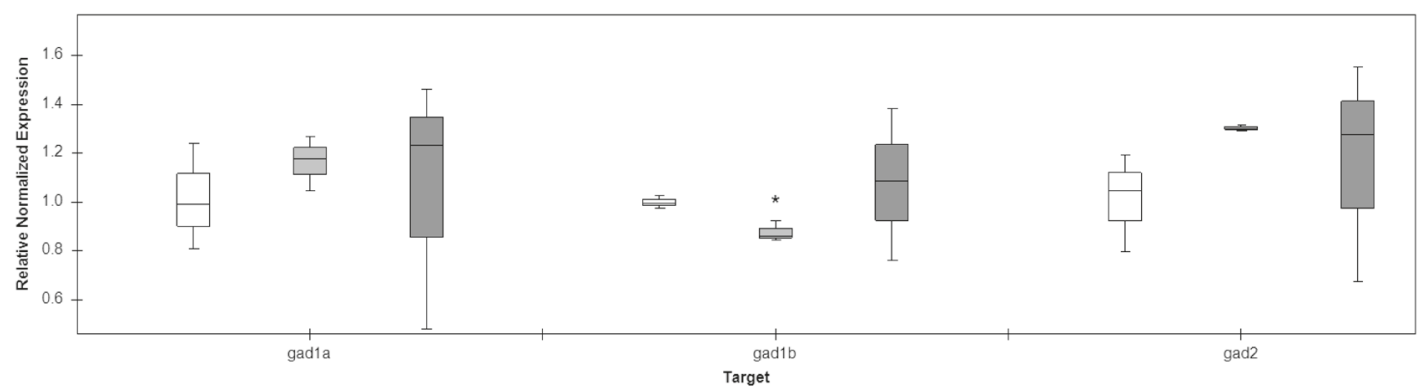

$\square+/+\quad \square+/-\quad \square$-/-

B

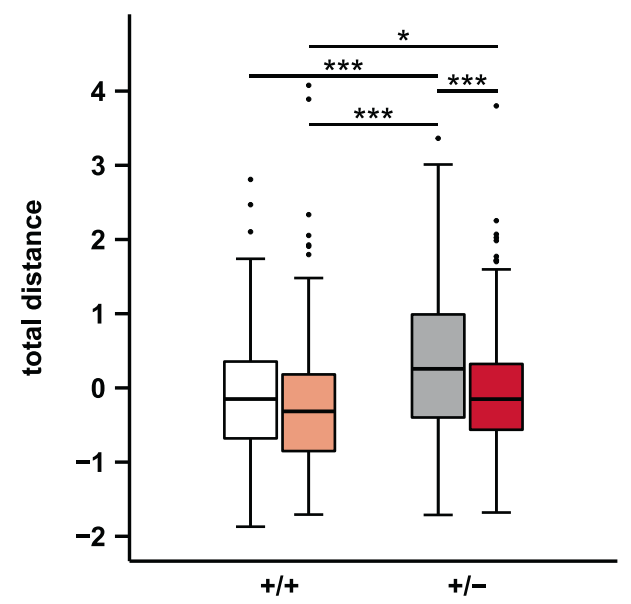

foxp $2^{+/+} n=195$

+ muscimol $n=187$

foxp $2^{+/-} n=213$

+ muscimol $n=210$

C

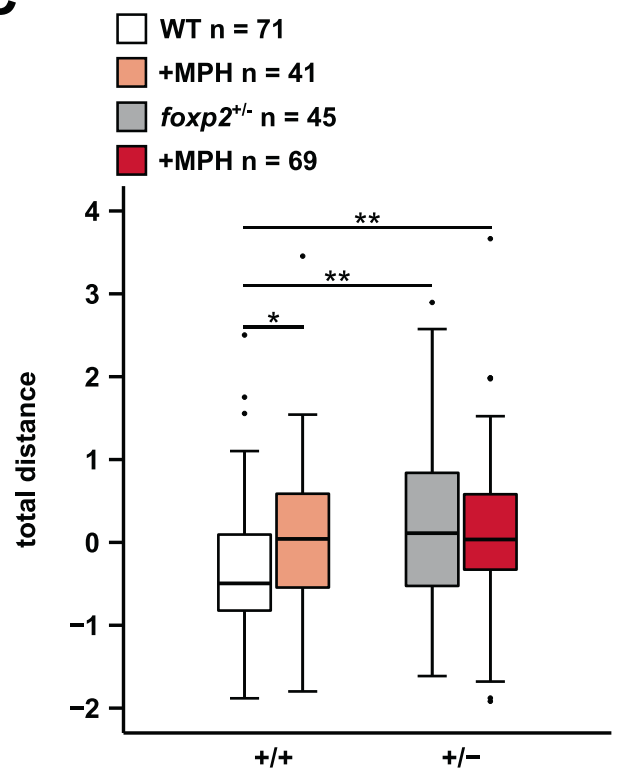

D

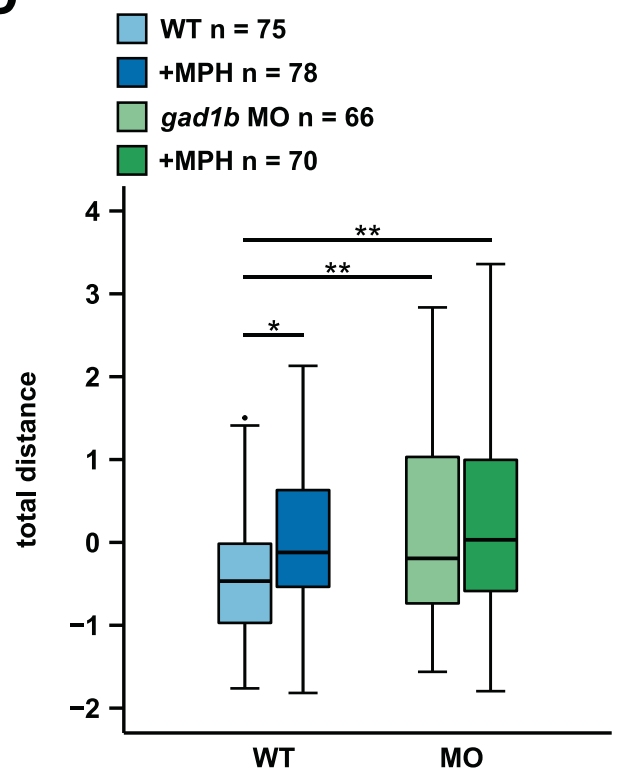

Fig. 5 Muscimol and methylphenidate differentially affects locomotor activity in foxp2 ${ }^{+/+}$and foxp2 $2^{+/-}$. A Transcript levels of gad1a, gad $1 b$ and gad 2 in foxp $2^{+/+}$(white), foxp $2^{+/-}$(light grey) and foxp2 $2^{-1-}$ (dark grey) siblings assessed by qPCR. ${ }^{*} P<0.05$. B Locomotor activity, displayed as total distance swum, of $5 \mathrm{dpf}$ old foxp $2^{+/+}$and foxp2 $2^{+/-}$following a bath-application in 0.05 mM GABA-A-R agonist muscimol (light red and dark red, respectively) or Danieau's solution (white and grey, respectively). C Locomotor activity, displayed as total distance swum, of $5 \mathrm{dpf}$ old foxp $2^{+/+}$and foxp $2^{+/-}$after exposure to Danieau's solution (white and grey, respectively) or 0.012 mM methylphenidate $(\mathrm{MPH}$, light red and dark red, respectively). D Locomotor activity, displayed as total distance swum, of $5 \mathrm{dpf}$ old gad1b morphants (MO) and wildtype controls (WT) exposed to Danieau's solution (light green and light blue, respectively) or $0.012 \mathrm{mM}$ MPH (dark green and dark blue, respectively). Raw data was standardised using $z$-score transformation. ${ }^{*} P<0.05,{ }^{* *} P<0.01,{ }^{* * * P}<0.001$. 
structural and functional striatal aberrations upon foxp2 loss-offunction $[19,25,28,29]$, our observations indicate that the effect may be rooted in the GABAergic neurons of the ventral forebrain. In conclusion, we provide experimental support for the hypothesis that FOXP2 is a likely player in the development of NDDs and that dysregulation of the GABAergic component impacting on the E/I balance should obtain increased attention in future research agendas addressing pathomechanism of NDDs.

\section{REFERENCES}

1. Demontis D, Walters RK, Martin J, Mattheisen M, Als TD, Agerbo E, et al. Discovery of the first genome-wide significant risk loci for attention deficit/hyperactivity disorder. Nat Genet. 2019;51:63-75.

2. Lai CSL, Fisher SE, Hurst JA, Vargha-Khadem F, Monaco AP. A forkhead-domain gene is mutated in a severe speech and language disorder. Nature. 2001:413:519-23.

3. Satterstrom FK, Kosmicki JA, Wang J, Breen MS, De Rubeis S, An JY, et al. Largescale exome sequencing study implicates both developmental and functional changes in the neurobiology of autism. Cell. 2020;180:568-584.

4. Herrero MJ, Gitton Y. The untold stories of the speech gene, the FOXP2 cancer gene. Genes Cancer. 2018;9:11-38.

5. Hickey SL, Berto S, Konopka G. Chromatin decondensation by FOXP2 promotes human neuron maturation and expression of neurodevelopmental disease genes. Cell Rep. 2019;27:1699-1711.

6. Co M, Anderson AG, Konopka G. FOXP transcription factors in vertebrate brain development, function, and disorders. WIREs Dev Biol. 2020;9:e375.

7. Bonkowsky JL, Chien C-B. Molecular cloning and developmental expression of foxP2 in zebrafish. Dev Dyn. 2005;234:740-6.

8. Campbell P, Reep RL, Stoll ML, Ophir AG, Phelps SM. Conservation and diversity of Foxp2 expression in muroid rodents: functional implications. J Comp Neurol. 2009;512:84-100.

9. Haesler S, Wada K, Nshdejan A, Morrisey EE, Lints T, Jarvis ED, et al. FoxP2 expression in avian vocal learners and non-learners. J Neurosci. 2004;24:3164-75.

10. Harvey-Girard E, Giassi ACC, Ellis W, Maler L. Organization of the gymnotiform fish pallium in relation to learning and memory: IV. Expression of conserved transcription factors and implications for the evolution of dorsal telencephalon. J Comp Neurol. 2012;520:3395-413.

11. Kato M, Okanoya $K$, Koike $T$, Sasaki $E$, Okano $H$, Watanabe $S$, et al. Human speechand reading-related genes display partially overlapping expression patterns in the marmoset brain. Brain Lang. 2014;133:26-38.

12. Mendoza E, Tokarev K, Düring DN, Retamosa EC, Weiss M, Arpenik N, et al. Differential coexpression of FoxP1, FoxP2, and FoxP4 in the Zebra Finch (Taeniopygia guttata) song system. J Comp Neurol. 2015;523:1318-40.

13. Pengra IGG, Marchaterre MA, Bass AH. FoxP2 expression in a highly vocal teleost fish with comparisons to tetrapods. Brain Behav Evol. 2018;91:82-96.

14. Rodenas-Cuadrado PM, Mengede J, Baas L, Devanna P, Schmid TA, Yartsev M, et al. Mapping the distribution of language related genes FoxP1, FoxP2, and CntnaP2 in the brains of vocal learning bat species. J Comp Neurol. 2018;526:1235-66.

15. Schön C, Wochnik A, Rößner A, Donow C, Knöchel W. The FoxP subclass in Xenopus laevis development. Dev Genes Evol. 2006;216:641-6.

16. Takahashi K, Liu FC, Oishi T, Mori T, Higo N, Hayashi M, et al. Expression of FOXP2 in the developing monkey forebrain: comparison with the expression of the genes FOXP1, PBX3, and MEIS2. J Comp Neurol. 2008;509:180-9.

17. Takahashi K, Liu F-C, Hirokawa K, Takahashi H. Expression of Foxp2, a gene involved in speech and language, in the developing and adult striatum. J Neurosci Res. 2003;73:61-72.

18. Castells-Nobau A, Eidhof I, Fenckova M, Brenman-Suttner DB, Scheffer-de Gooyert $\mathrm{JM}$, Christine $\mathrm{S}$, et al. Conserved regulation of neurodevelopmental processes and behavior by FoxP in Drosophila. PLoS ONE. 2019;14:e0211652.

19. Chen Y-C, Kuo HY, Bornschein U, Takahashi H, Chen SY, Lu KM, et al. Foxp2 controls synaptic wiring of corticostriatal circuits and vocal communication by opposing Mef2c. Nat Neurosci. 2016;19:1513-22.

20. Chiu Y-C, Li MY, Liu YH, Ding JY, Yu JY, Wang TW. Foxp2 regulates neuronal differentiation and neuronal subtype specification. Dev Neurobiol. 2014;74:723-38.

21. Clovis YM, Enard W, Marinaro F, Huttner WB, Tonelli DDP. Convergent repression of Foxp2 3'UTR by miR-9 and miR-132 in embryonic mouse neocortex: implications for radial migration of neurons. Development. 2012;139:3332-42.

22. Enard W, Gehre S, Hammerschmidt K, Hölter SM, Blass T, Somel M, et al. A humanized version of Foxp2 affects cortico-basal ganglia circuits in mice. Cell. 2009;137:961-71.

23. French CA, Jin X, Campbell TG, Gerfen E, Groszer M, Fisher SE, et al. An aetiological Foxp2 mutation causes aberrant striatal activity and alters plasticity during skill learning. Mol Psychiatry. 2012;17:1077-85.
24. Groszer M, Keays DA, Deacon RM, de Bono JP, Prasad-Mulcare S, Gaub S, et al. Impaired synaptic plasticity and motor learning in mice with a point mutation implicated in human speech deficits. Curr Biol. 2008;18:354-62.

25. Schulz SB, Haesler S, Scharff C, Rochefort C. Knockdown of FoxP2 alters spine density in Area $X$ of the zebra finch. Genes Brain Behav. 2010;9:732-40.

26. Shu W, Cho JY, Jiang Y, Zhang M, Weisz D, Elder GA, et al. Altered ultrasonic vocalization in mice with a disruption in the Foxp2 gene. Proc Natl Acad Sci. 2005;102:9643-8.

27. Tsui $D$, Vessey JP, Tomita $H$, Kaplan DR, Miller FD. FoxP2 regulates neurogenesis during embryonic cortical development. J Neurosci. 2013;33:244-58.

28. Vernes SC, Oliver PL, Spiteri E, Lockstone HE, Puliyadi R, Taylor JM, et al. Foxp2 regulates gene networks implicated in neurite outgrowth in the developing brain. PLoS Genet. 2011;7:e1002145.

29. van Rhijn J-R, Fisher SE, Vernes SC, Nadif Kasri N. Foxp2 loss of function increases striatal direct pathway inhibition via increased GABA release. Brain Struct Funct. 2018;223:4211-26.

30. Sohal VS, Rubenstein JLR. Excitation-inhibition balance as a framework for investigating mechanisms in neuropsychiatric disorders. Mol Psychiatry. 2019;24:1248-57.

31. $\mathrm{He} \mathrm{H}$, Cline HT. What is excitation/inhibition and how is it regulated? A case of the elephant and the wisemen. J Exp Neurosci. 2019;13:1179069519859371.

32. Gao R, Penzes P. Common mechanisms of excitatory and inhibitory imbalance in schizophrenia and autism spectrum disorders. Curr Mol Med. 2015;15:146-67.

33. Hayes DJ, Jupp B, Sawiak SJ, Merlo E, Caprioli D, Dalley JW. Brain Y-aminobutyric acid: a neglected role in impulsivity. Eur J Neurosci. 2014;39:1921-32.

34. Rivero O, Selten MM, Sich S, Popp S, Bacmeister L, Amendola E, et al. Cadherin-13, a risk gene for ADHD and comorbid disorders, impacts GABAergic function in hippocampus and cognition. Transl Psychiatry. 2015;5:e655.

35. Mossink B, van Rhijn JR, Wang S, Linda K, Vitale MR, Zöller J, et al. Cadherin-13 is a critical regulator of GABAergic modulation in human stem cell derived neuronal networks. Mol Psychiatry. 10 May 2021. https://doi.org/10.1038/s41380-021-01117-x.

36. Serrano-Regal MP, Luengas-Escuza I, Bayón-Cordero L, Ibarra-Aizpurua N, Alberd $E$, Pérez-Samartín $A$, et al. Oligodendrocyte differentiation and myelination is potentiated via GABAB receptor activation. Neuroscience 2020;439:163-80.

37. Huang ZJ, Paul A. The diversity of GABAergic neurons and neural communication elements. Nat Rev Neurosci. 2019;20:563-72.

38. Polan MB, Pastore MT, Steingass K, Hashimoto S, Thrush DL, Pyatt R, et al. Neurodevelopmental disorders among individuals with duplication of $4 \mathrm{p} 13$ to $4 \mathrm{p} 12$ containing a $\mathrm{GABA}_{\mathrm{A}}$ receptor subunit gene cluster. Eur J Hum Genet. 2014;22:105-9.

39. Bruxel EM, Akutagava-Martins GC, Salatino-Oliveira A, Genro JP, Zeni CP, Polanczyk GV, et al. GAD1 gene polymorphisms are associated with hyperactivity in attention-deficit/hyperactivity disorder. Am J Med Genet B Neuropsychiatr Genet. 2016;171:1099-104.

40. Yuan F, Gu X, Huang X, Zhong Y, Wu J. SLC6A1 gene involvement in susceptibility to attention-deficit/hyperactivity disorder: a case-control study and gene-environment interaction. Prog Neuropsychopharmacol Biol Psychiatry. 2017;77:202-8.

41. Naaijen J, Bralten J, Poelmans G, IMAGE C, Glennon JC, Franke B, et al. Glutamatergic and GABAergic gene sets in attention-deficit/hyperactivity disorder: association to overlapping traits in ADHD and autism. Transl Psychiatry. 2017;7:e999.

42. Chen L, Yang X, Zhou X, Wang C, Gong X, Chen B, et al. Hyperactivity and impaired attention in Gamma aminobutyric acid transporter subtype 1 gene knockout mice. Acta Neuropsychiatr. 2015;27:368-74.

43. Miyata S, Kakizaki T, Fujihara K, Obinata H, Hirano T, Nakai J, et al. Global knockdown of glutamate decarboxylase 67 elicits emotional abnormality in mice. Mol Brain. 2021;14:5.

44. Schuler V, Lüscher C, Blanchet C, Klix N, Sansig G, Klebs K, et al. Epilepsy, hyperalgesia, impaired memory, and loss of pre- and postsynaptic GABAB responses in mice lacking GABAB(1). Neuron. 2001;31:47-58.

45. Yang P, Cai G, Cai Y, Fei J, Liu G. Gamma aminobutyric acid transporter subtype 1 gene knockout mice: a new model for attention deficit/hyperactivity disorder. Acta Biochim Biophys Sin. 2013;45:578-85.

46. Faraone SV, Asherson P, Banaschewski T, Biederman J, Buitelaar JK, RamosQuiroga JA, et al. Attention-deficit/hyperactivity disorder. Nat Rev Dis Prim. 2015;1:15020.

47. Bollmann S, Ghisleni C, Poil SS, Martin E, Ball J, Eich-Höchli D, et al. Developmental changes in gamma-aminobutyric acid levels in attention-deficit/hyperactivity disorder. Transl Psychiatry. 2015;5:e589.

48. Edden RAE, Crocetti D, Zhu H, Gilbert DL, Mostofsky SH. Reduced GABA concentration in attention-deficit/hyperactivity disorder. Arch Gen Psychiatry. 2012;69:750-3

49. Puts NA, Ryan M, Oeltzschner G, Horska A, Edden R, Mahone EM. Reduced striatal GABA in unmedicated children with ADHD at 7T. Psychiatry Res Neuroimaging. 2020;301:111082.

50. Chen Y-C, Sudre G, Sharp W, Donovan F, Chandrasekharappa SC, Hansen N, et al. Neuroanatomic, epigenetic and genetic differences in monozygotic twins discordant for attention deficit hyperactivity disorder. Mol Psychiatry. 2018;23:683-90. 
51. Kimmel CB, Ballard WW, Kimmel SR, Ullmann B, Schilling TF. Stages of embryonic development of the zebrafish. Dev Dyn. 1995;203:253-310.

52. Thisse $C$, Thisse B. High-resolution in situ hybridization to whole-mount zebrafish embryos. Nat Protoc. 2008;3:59-69.

53. Stehr CM, Linbo TL, Incardona JP, Scholz NL. The developmental neurotoxicity of Fipronil: notochord degeneration and locomotor defects in zebrafish embryos and larvae. Toxicol Sci. 2006;92:270-8.

54. Lange $M$, Norton $W$, Coolen $M$, Chaminade $M$, Merker $S$, Proft $F$, et al. The ADHDsusceptibility gene Iphn3.1 modulates dopaminergic neuron formation and locomotor activity during zebrafish development. Mol Psychiatry. 2012;17:946-54.

55. Faul F, Erdfelder E, Buchner A, Lang A-G. Statistical power analyses using G*Power 3.1: tests for correlation and regression analyses. Behav Res Methods. 2009;41:1149-60.

56. Vernes SC, Nicod J, Elahi FM, Coventry JA, Kenny N, Coupe AM, et al. Functional genetic analysis of mutations implicated in a human speech and language disorder. Hum Mol Genet. 2006;15:3154-67.

57. Vernes SC, Newbury DF, Abrahams BS, Winchester L, Nicod J, Groszer M, et al. A functional genetic link between distinct developmental language disorders. N. Engl J Med. 2008;359:2337-45.

58. French CA, Fisher SE. What can mice tell us about Foxp2 function? Curr Opin Neurobiol. 2014;28:72-9.

59. Xing L, Hoshijima K, Grunwald DJ, Fujimoto E, Quist TS, Sneddon J, et al. Zebrafish foxP2 zinc finger nuclease mutant has normal axon pathfinding. PLoS ONE. 2012;7:e43968.

60. Hoffman EJ, Turner KJ, Fernandez JM, Cifuentes D, Ghosh M, ljaz S, et al. Estrogens suppress a behavioral phenotype in zebrafish mutants of the autism risk gene, CNTNAP2. Neuron. 2016;89:725-33.

61. Vargha-Khadem F, Gadian DG, Copp A, Mishkin M. FOXP2 and the neuroanatomy of speech and language. Nat Rev Neurosci. 2005;6:131-8.

62. French CA, Vinueza Veloz MF, Zhou K, Peter S, Fisher SE, Costa RM, et al. Differential effects of Foxp2 disruption in distinct motor circuits. Mol Psychiatry. 2019:24:447-62.

63. Haesler S, Rochefort C, Georgi B, Licznerski P, Osten P, Scharff C. Incomplete and inaccurate vocal imitation after knockdown of FoxP2 in songbird basal ganglia nucleus Area X. PLOS Biol. 2007;5:e321.

64. Murugan M, Harward S, Scharff C, Mooney R. Diminished FoxP2 levels affect dopaminergic modulation of corticostriatal signaling important to song variability. Neuron. 2013;80:1464-76.

65. Grillner S, El Manira A. Current principles of motor control, with special reference to vertebrate locomotion. Physiol Rev. 2019;100:271-320.

66. Heusner CL, Beutler LR, Houser CR, Palmiter RD. Deletion of GAD67 in dopamine receptor-1 expressing cells causes specific motor deficits. Genes N. Y N. 2000. 2008:46:357-67.

67. Rossi A, Kontarakis Z, Gerri C, Nolte H, Hölper S, Krüger M, et al. Genetic compensation induced by deleterious mutations but not gene knockdowns. Nature. 2015;524:230-3.

68. Araujo DJ, Anderson AG, Berto S, Runnels W, Harper M, Ammanuel S, et al. FoxP1 orchestration of ASD-relevant signaling pathways in the striatum. Genes Dev. 2015;29:2081-96.

69. Anderson AG, Kulkarni A, Harper M, Konopka G. Single-cell analysis of Foxp1-driven mechanisms essential for striatal development. Cell Rep. 2020;30:3051-3066.e7.

70. Arcos-Burgos M, Jain M, Acosta MT, Shively S, Stanescu H, Wallis D, et al. A common variant of the latrophilin 3 gene, LPHN3, confers susceptibility to ADHD and predicts effectiveness of stimulant medication. Mol Psychiatry. 2010;15:1053-66.

71. Chandley MJ, Crawford JD, Szebeni A, Szebeni K, Ordway GA. NTRK2 expression levels are reduced in laser captured pyramidal neurons from the anterior cingulate cortex in males with autism spectrum disorder. Mol Autism. 2015;6:28.

72. Mueller $T$, Vernier $P$, Wullimann MF. A phylotypic stage in vertebrate brain development: GABA cell patterns in zebrafish compared with mouse. J Comp Neurol. 2006;494:620-34

73. Rivero O, Sich S, Popp S, Schmitt A, Franke B, Lesch KP. Impact of the ADHDsusceptibility gene $\mathrm{CDH} 13$ on development and function of brain networks. Eur Neuropsychopharmacol. 2013;23:492-507.

74. Peñagarikano $O$, Abrahams BS, Herman El, Winden KD, Gdalyahu A, Dong $H$, et al. Absence of CNTNAP2 leads to epilepsy, neuronal migration abnormalities, and core autism-related deficits. Cell. 2011;147:235-46.

75. Freese L, Muller EJ, Souza MF, Couto-Pereira NS, Tosca CF, Ferigolo M, et al. GABA system changes in methylphenidate sensitized female rats. Behav Brain Res. 2012:231:181-6.

76. Goitia B, Raineri M, González LE, Rozas JL, Garcia-Rill E, Bisagno V, et al. Differential effects of methylphenidate and cocaine on GABA transmission in sensory thalamic nuclei. J Neurochem. 2013;124:602-12.

77. Solleveld MM, Schrantee A, Puts NAJ, Reneman L, Lucassen PJ. Age-dependent, lasting effects of methylphenidate on the GABAergic system of ADHD patients. Neurolmage Clin. 2017;15:812-8.
78. Askenasy EP, Taber KH, Yang PB, Dafny N. Methylphenidate (ritalin): behavioral studies in the rat. Int J Neurosci. 2007;117:757-94.

79. Souza BR, Romano-Silva MA, Tropepe V. Dopamine D2 receptor activity modulates Akt signaling and alters GABAergic neuron development and motor behavior in zebrafish larvae. J Neurosci. 2011;31:5512-25.

80. Co M, Hickey SL, Kulkarni A, Harper M, Konopka G. Cortical Foxp2 supports behavioral flexibility and developmental dopamine D1 receptor expression. Cereb Cortex. 2020;30:1855-70.

81. Moreno N, González A, Rétaux S. Development and evolution of the subpallium. Semin Cell Dev Biol. 2009;20:735-43.

\section{ACKNOWLEDGEMENTS}

We thank Manfred Schartl, Eva Klopocki and Thomas Haaf for providing laboratory space, Zuzana Fouskova and the animal caretakers for technical assistance and Daniel Liedtke, Mateus Adolfi, past and present lab members for support and discussions. Christine Krempl and the CoreUnit for Confocal Microscopy at the Institute for Virology and Immune Biology for access to and support with confocal imaging. TML and AD are members of the Graduate School of Life Science (GSLS, University of Würzburg).

\section{AUTHOR CONTRIBUTIONS}

$T M L, M R, C D$ and $C L$ conceived the study. TML, CD and $C L$ designed the experiments. $T M L$ and $A D$ performed the experiments with the support from $M B, Z G, V S, C D$ and $C L$. TML, AD, MB, ZG, VS, CD and $C L$ analysed the data. TML, CD and $C L$ wrote the manuscript. All authors participated in interpretation of results, revised and edited the manuscript. MR, $C D$ and $C L$ acquired funding and administered the project.

\section{FUNDING}

This project was funded by the Interdisciplinary Center for Clinical Research (Interdisziplinäres Zentrum für Klinische Forschung, Universitätsklinikum Würzburg, IZKF) of University of Würzburg (project number N-320 and Z-4/149), the Verein zur Durchführung Neurowissenschaftlicher Tagungen e.V. and the Faculty of Biology, University of Würzburg. This publication was supported by the Open Access Publication Fund of the University of Würzburg. Open Access funding enabled and organized by Projekt DEAL.

\section{COMPETING INTERESTS}

The authors declare no competing interests.

\section{ADDITIONAL INFORMATION}

Supplementary information The online version contains supplementary material available at https://doi.org/10.1038/s41398-021-01651-w.

Correspondence and requests for materials should be addressed to Christina Lillesaar.

Reprints and permission information is available at http://www.nature.com/ reprints

Publisher's note Springer Nature remains neutral with regard to jurisdictional claims in published maps and institutional affiliations. Attribution 4.0 International License, which permits use, sharing, adaptation, distribution and reproduction in any medium or format, as long as you give appropriate credit to the original author(s) and the source, provide a link to the Creative Commons license, and indicate if changes were made. The images or other third party material in this article are included in the article's Creative Commons license, unless indicated otherwise in a credit line to the material. If material is not included in the article's Creative Commons license and your intended use is not permitted by statutory regulation or exceeds the permitted use, you will need to obtain permission directly from the copyright holder. To view a copy of this license, visit http://creativecommons. org/licenses/by/4.0/

(c) The Author(s) 2021 